\title{
RAD18 contributes to the migration and invasion of human cervical cancer cells via the interleukin-1 $\beta$ pathway
}

\author{
PENGRONG LOU ${ }^{1 *}$, SHITAO ZOU ${ }^{2 *}$, ZENGFU SHANG ${ }^{3 *}, \mathrm{CHAO} \mathrm{HE}^{2}$, \\ AIDI GAO ${ }^{2}$, SHUNYU HOU ${ }^{4}$ and JUNDONG ZHOU ${ }^{2}$ \\ ${ }^{1}$ Center of Chemoradio-Oncology, Ningbo First Hospital, Ningbo, Zhejiang 315010; ${ }^{2}$ Suzhou Cancer Center Core Laboratory, \\ Nanjing Medical University Affiliated Suzhou Hospital, Suzhou, Jiangsu 215001; ${ }^{3}$ School of Radiation Medicine \\ and Protection, Medical College of Soochow University, Collaborative Innovation Center of Radiation Medicine \\ of Jiangsu Higher Education Institutions, Suzhou, Jiangsu 215123; ${ }^{4}$ Department of Gynecology, \\ Nanjing Medical University Affiliated Suzhou Hospital, Suzhou, Jiangsu 215001, P.R. China
}

Received March 20, 2019; Accepted July 11, 2019

DOI: $10.3892 / \mathrm{mmr} .2019 .10564$

\begin{abstract}
The E3 ubiquitin ligase RAD18 has been identified as an oncoprotein that exhibits prometastatic properties in various types of cancer; however, the role of RAD18 in cervical cancer (CC) remains unclear. In the present study, it was revealed that increased expression of RAD18 was associated with worse prognosis of patients with CC. Knockdown of endogenous RAD18 suppressed the motility and invasiveness of CC cells, as evaluated by Transwell assays. mRNA sequencing revealed that silencing RAD18 altered the expression profile of proinflammatory mediators, such as interleukin-1 $\beta$ (IL-1 $\beta$ ). Furthermore, exogenous IL-1 $\beta$ treatment rescued RAD18-mediated $\mathrm{CC}$ cell invasion. These findings indicated an underlying mechanism via which RAD18 promotes $\mathrm{CC}$ progression, suggesting that RAD18 may be a potential biomarker and therapeutic target for malignant $\mathrm{CC}$.
\end{abstract}

\section{Introduction}

Cervical cancer (CC) is the fourth most common malignant tumor and a leading cause of cancer-associated mortality in women, accounting for nearly 265,700 deaths globally in 2012 (1) Despite advances in early diagnosis, surgical resection, and radio- and chemotherapy, the overall survival (OS)

Correspondence to: Professor Shunyu Hou, Department of Gynecology, Nanjing Medical University Affiliated Suzhou Hospital, A16 Baita West Road, Suzhou, Jiangsu 215001, P.R. China E-mail: houshunyu@sina.com

Professor Jundong Zhou, Suzhou Cancer Center Core Laboratory, Nanjing Medical University Affiliated Suzhou Hospital, 16 Baita West Road, Suzhou, Jiangsu 215001, P.R. China

E-mail: zhoujundong330@163.com

*Contributed equally

Key words: cervical cancer, RAD18, metastasis, interleukin-1 $\beta$ rate of patients with $\mathrm{CC}$ remains low, particularly for patients with metastasis (2). The five-year survival rate of patients decreased from 85 to $50 \%$ for patients with lymph node metastasis compared with patients with cervical carcinoma in situ (3). The median OS of patients with bone metastasis was only 23 months (4). Consequently, identifying the molecular events involved in the invasion and metastasis of $\mathrm{CC}$ is required in order to develop novel therapeutic strategies and improve $\mathrm{CC}$ outcomes.

Cellular DNA is continuously exposed to a range of endogenous and exogenous damaging factors, which contribute to genome instability and are thus associated with carcinogenesis or cell death (5). If DNA damage occurs during replication, it will result in replication fork stalling, subsequently leading to the collapse of the replication fork and genome rearrangements (6). To avoid the occurrence of severe aberrations of the genome, cells have evolved a translesion DNA synthesis (TLS) system that employs a series of specialized DNA polymerases with low fidelity to replicate damaged DNA templates and relieve the DNA replication stress (7). Various DNA polymerases (pols), including pol $\mathbf{t}$, pol $\kappa$ and pol $\eta$, belong to the Y-family, whereas pol $\zeta$ is a B-family polymerase (8). Due to the high error-proneness of these TLS polymerases, their recruitment to DNA is tightly regulated via several mechanisms. For example, proliferating cell nuclear antigen (PCNA), as a scaffold for replicative polymerases, is monoubiquitylated at Lys164 in mammalian cells when exposed to various DNA lesion-inducing agents, subsequently stalling the replication fork and mediating the access of TLS polymerases (9). The E3 ubiquitin ligase RAD18 forms a E2-E3 complex with human homolog of RAD6 (hHR6)A/hHR6B and facilitates PCNA monoubiquitination in human cells $(10,11)$.

The TLS system is a double-edged sword in guarding the genome. It has been reported that low-fidelity DNA polymerases are involved in the accumulation of spontaneous and DNA damage-induced genomic mutations, which are recognized as a driving force for malignant transformation (12). As a key regulator of the TLS pathway, RAD18 has been identified as an oncoprotein that exhibits elevated expression in primary 
and metastatic melanoma (13). Increased expression of RAD18 is associated with worse 5-year survival in patients with melanoma (13). Zhou et al (14) revealed that RAD18 expression was significantly increased in esophageal squamous cell cancer (ESCC) tissue compared with adjacent normal tissues. A subsequent study by the same group demonstrated that RAD18 expression levels were inversely associated with patient survival, and that RAD18 contributed to ESCC cell proliferation and migration by activating the JNK-matrix metalloproteinase (MMP) pathway (15).

In the present study, the role of RAD18 in CC progression was investigated. It was demonstrated that upregulated expression of RAD18 was associated with worse patient prognosis. RNA sequencing (RNA-Seq) was performed in CC cells following RAD18 knockdown, revealing that silencing RAD18 altered the expression profile of proinflammatory mediators, such as interleukin-1 $\beta$ (IL-1 $\beta$ ). Furthermore, exogenous IL-1 $\beta$ treatment rescued RAD18-mediated CC cell invasion following RAD18 knockdown.

\section{Materials and methods}

Tissue samples and cell lines. A total of 126 archival paraffin-embedded human CC tissues (29-70 years) were used in the present study. The samples were collected at the Nanjing Medical University Affiliated Suzhou Hospital between January 2010 and November 2011. Informed patient consent was obtained at the time of collection. The clinicopathological features of the patients were staged according to the 8th Union of International Control of Cancer (UICC) classification (16) and presented in Table I. The use of the tissues for the present retrospective study was approved by the Institutional Ethics Committee of the Nanjing Medical University. The human $\mathrm{CC}$ cell lines HeLa229 and SiHa were purchased from the Shanghai Cell Bank. Cells were cultured in DMEM (HyClone; GE Healthcare Life Sciences) supplemented with $10 \%$ FBS (HyClone; GE Healthcare Life Sciences) and antibiotics (100 $\mu \mathrm{g} / \mathrm{ml}$ streptomycin and $100 \mathrm{U} / \mathrm{ml}$ penicillin; Corning Inc.), and incubated in humidified atmosphere with $5 \% \mathrm{CO}_{2}$ at $37^{\circ} \mathrm{C}$.

Immunohistochemical analyses. The expression levels of RAD18 in human CC tissues were detected using immunohistochemistry. CC tissues (4- $\mu \mathrm{m}$ sections) were deparaffinized and heat-treated at $100^{\circ} \mathrm{C}$ with citrate buffer for antigen retrieval, and then incubated $0.03 \%$ hydrogen peroxide for 5 min to block endogenous peroxidases. Following blocking with $2 \%$ bovine serum albumin (Sigma-Aldrich; Merck KGaA) at $37^{\circ} \mathrm{C}$ for $30 \mathrm{~min}$, the tissues were incubated with anti-RAD18 antibody (1:200; cat. no. ab186835; Abcam) at $37^{\circ} \mathrm{C}$ for $2 \mathrm{~h}$. Horseradish peroxidase (HRP)-conjugated anti-rabbit antibody (1:200; cat. no. GP021729; Shanghai GeneTech Co., Ltd.) was then added for $1 \mathrm{~h}$ at $37^{\circ} \mathrm{C}$, and color was developed using 3,3'-diaminobenzidine. The tissue sections were counterstained with hematoxylin at room temperature for $5 \mathrm{~min}$ and washed briefly in a water bath containing drops of ammonia, prior to dehydration and mounting in Diatex. RAD18 expression levels in three randomly selected fields per sample were scored using a light microscope (magnification, x100; Leica Microsystems $\mathrm{GmbH}$ ) as previously described (15) and defined by the final score as follows: Low expression (score $\leq 6$, $\mathrm{n}=81$ ) and high expression (score $>6, \mathrm{n}=45$ ).

Stable cell line generation. Short hairpin RNA (shRNA) targeting RAD18 (shRAD18: 5'-GCTGTTTATCACGCG AAGA-3') or non-targeting negative control shRNA (shNC: 5'-TTCTCCGAACGTGTCACGT-3') were purchased from Guangzhou RiboBio Co., Ltd. The fragments were inserted into a lentiviral expression vector containing EGFP (pGLVH1/GFP+Puro; Shanghai GenePharma Co., Ltd.) and packaged into viral particles by Shanghai GenePharma Co., Ltd. A total of $2 \times 10^{6} \mathrm{TU}$ of virus particles were mixed with $5 \mu \mathrm{g} / \mathrm{ml}$ polybrene in $500 \mu \mathrm{l}$ DMEM, then added to $5 \times 10^{4} \mathrm{HeLa} 229$ cells and SiHa cells in 24 -well plates. The cells were then selected in medium containing $1 \mu \mathrm{g} / \mathrm{ml}$ Puromycin (Sigma-Aldrich; Merck KGaA) for 1 week. The stable cell lines were validated via western blotting.

Western blot analysis. Cells were harvested and lysed in mammalian protein extraction reagent (Thermo Fisher Scientific, Inc.) containing protease inhibitors (Thermo Fisher Scientific, Inc.) for $10 \mathrm{~min}$ on ice. The protein concentrations were measured using a bicinchoninic acid protein assay kits (Thermo Fisher Scientific, Inc.), and $20 \mu \mathrm{g} / \mathrm{lane}$ protein was separated via 10\% SDS-PAGE and transferred to PVDF membranes (EMD Millipore). The membranes were blocked with $5 \%$ nonfat milk at room temperature for $1 \mathrm{~h}$ and incubated with primary antibodies targeting RAD18 (1:1,000; cat. no. ab186835; Abcam) or $\beta$-actin $(1: 1,000$; cat. no. AA128; Beyotime Institute of Biotechnology) overnight at $4^{\circ} \mathrm{C}$. The membranes were washed and then incubated with HRP-conjugated anti-rabbit (1:2,000; cat. no. A0208) or anti-mouse secondary antibodies (1:2,000; cat. no. A0216; Beyotime Institute of Biotechnology) at room temperature for $1 \mathrm{~h}$. The protein bands were visualized using High-sig ECL Western Blotting Substrate (Tanon Science and Technology Co., Ltd.).

$R N A$ extraction and reverse transcription-quantitative $P C R(R T-q P C R)$. Total RNA was isolated from cells using TRIzol ${ }^{\circledR}$ reagent (Invitrogen; Thermo Fisher Scientific, Inc.) according to the manufacturer's protocols. The concentrations of RNA were determined using a NanoDrop 2000 (Nanodrop Technologies; Thermo Fisher Scientific, Inc.). RNA (500 ng) from each sample was subjected to RT using a RevertAid First Strand cDNA Synthesis kit (Thermo Fisher Scientific, Inc.) at $60^{\circ} \mathrm{C}$ for $5 \mathrm{~min}, 42^{\circ} \mathrm{C}$ for $50 \mathrm{~min}$ and $70^{\circ} \mathrm{C}$ for $15 \mathrm{~min}$ ). The mRNA levels of target genes were measured via qPCR using QuantiFast SYBR ${ }^{\circledR}$ Green PCR kit (Qiagen, Inc.) on a StepOne Plus instrument (Applied Biosystems; Thermo Fisher Scientific, Inc.) under the following conditions: $95^{\circ} \mathrm{C}$ for $2 \mathrm{~min}$, then 20 or 40 cycles of $95^{\circ} \mathrm{C}$ for $15 \mathrm{sec}$ and $60^{\circ} \mathrm{C}$ for $60 \mathrm{sec}$. The PCR products of 20 cycles were separated via electrophoresis on a $1.5 \%$ agarose gel and stained with ethidium bromide, then visualized using Bio-Rad ChemiDoc MP (Bio-Rad Laboratories, Inc.) and analyzed using Image Lab 5.2.1 software (Bio-Rad Laboratories, Inc.). The primers are listed in Table II. Relative expression levels were calculated using the $2^{-\Delta \Delta \mathrm{Cq}}(17)$ method and normalized to $\beta$-actin expression levels. 
Table I. Clinicopathological parameters of patients with cervical cancer.

\begin{tabular}{lr}
\hline Clinicopathological parameters & Cases \\
\hline Age (years) & \\
$<50$ & 84 \\
$\geq 50$ & 42 \\
T stage & \\
T1 & 69 \\
T2-T4 & 57 \\
N stage & \\
N0 & 101 \\
N1 & 25 \\
Clinical stage & \\
I & 69 \\
II-IV & 57 \\
\hline
\end{tabular}

Table II. Primer sequences for reverse transcription-quantitative PCR analysis.

\begin{tabular}{ll}
\hline Genes & \multicolumn{1}{c}{ Primers } \\
\hline BIRC3 & 5'-AGTTCATCCGTCAAGTT-3' sense \\
& 5'-ATCTCCTGGGCTGTC-3' antisense \\
CXCL2 & 5'-TGCGCTGCCAGTGCTTG-3' sense \\
& 5'-TGGCTATGACTTCGGTTTGG-3' antisense \\
CXCL8 & 5'-GCCTTCCTGATTTCTGC-3' sense \\
& 5'-CCCTCTGCACCCAGTT-3' antisense \\
CXCL16 & 5'-AGATGGGACGGGACTTGC-3' sense \\
& 5'-GGAGCTGGAACCTCGTGTAG-3' antisense \\
EGR1 & 5'-CACGAACGCCCTTACGC-3' sense \\
& 5'-TTCATCGCTCCTGGCAAAC-3' antisense \\
ICAM1 & 5'-TGCAAGAAGATAGCCAACCA-3' sense \\
& 5'-GCCAGTTCCACCCGTTC-3' antisense \\
IL-1 $\alpha$ & 5'-GCCAAAGTTCCAGACA-3' sense \\
& 5'-GGTTGCTACTACCACCA-3' antisense \\
IL-1 $\beta$ & 5'-ACAGTGGCAATGAGGATG-3' sense \\
& 5'-TGTAGTGGTGGTCGGAGA-3' antisense \\
IL-6 & 5'-CCCTGAGAAAGGAGACA-3' sense \\
& 5'-CAAATCTGTTCTGGAGGT-3' antisense \\
STAT1 & 5'-TGAACTTACCCAGAATGCC-3' sense \\
& 5'-TGCTGCCGAACTTGC-3' antisense \\
& 5'-AGCGAGCATCCCCCAAAGTT-3' sense \\
& \\
& \\
&
\end{tabular}

BIRC3, baculoviral IAP repeat containing 3; CXCL, C-X-C motif chemokine ligand; EGR1, early growth response 1; ICAM1, intercellular adhesion molecule 1; IL, interleukin.

RNA-Seq transcriptome analysis. Total RNA from $\mathrm{SiHa}$ $\mathrm{shNC} / \mathrm{shRAD} 18$ was prepared and stored at $-80^{\circ} \mathrm{C}$. The RNA quality was determined using a Bioanalyzer 2200 (Agilent Technologies, Inc.), and an RNA integrity number $>8.0$ was considered acceptable for cDNA library construction. Sequencing and bioinformatic analysis were performed by Vazyme Biotech Co., Ltd. Gene expression quantification was performed using Cufflinks 2.2.1 (http://cole-trapnell-lab. github.io/cufflinks/releases/v2.2.1/). Genes were considered to be significantly differentially expressed between groups when $\mathrm{P}<0.05$ and the fold change of expression was $>1.5$. Gene Ontology (GO) and pathway analyses were performed using the GO (http://geneontology.org/) and Kyoto Encyclopedia of Genes and Genomes (KEGG) databases (https://www.genome. jp/kegg/kegg1.html).

Cell migration and invasion analysis. Cell migration was analyzed using Transwell chambers (Corning Inc.). Cells [5x $10^{4}$; treated with/without $10 \mathrm{ng} / \mathrm{ml} \mathrm{IL-1 \beta}$ (PeproTech, Inc.) for $24 \mathrm{~h}$ ] were suspended in $200 \mu \mathrm{l}$ serum-free medium and seeded in the upper chamber. The lower chambers were filled with $600 \mu \mathrm{l}$ media containing $10 \%$ FBS. Following incubation for $24 \mathrm{~h}$, the cells remaining in the upper chambers were scraped off, and the migrating cells were fixed with $3.7 \%$ paraformaldehyde and stained with Giemsa (both at room temperature for $5 \mathrm{~min}$ ), then imaged under a light microscope (magnification, x100; Leica Microsystems $\mathrm{GmbH}$ ) in three randomly selected fields per sample.

Cell invasion was assessed using Matrigel-coated Transwell chambers (Corning Inc.). A total of $1 \times 10^{5}$ cells treated with/without IL-1 $\beta$ (10 $\mathrm{ng} / \mathrm{ml})$ for $24 \mathrm{~h}$ were suspended in $200 \mu \mathrm{l}$ serum-free media and plated in the upper chamber, which was pre-coated with $60 \mu$ l Matrigel (1:6 dilution; BD Biosciences). The lower chambers were filled with $600 \mu \mathrm{l}$ media containing 10\% FBS. Following incubation for $48 \mathrm{~h}$, the cells that invaded through the Matrigel to the lower surface of the filter were fixed with $3.7 \%$ paraformaldehyde and stained with Giemsa as aforementioned. The penetration of cells through the membrane was determined under a light microscope (magnification, x 100; Leica Microsystems GmbH) in three randomly selected fields per sample.

Statistical analysis. Data are presented as mean \pm standard deviation $(n=3)$. Statistical analyses were performed using SPSS 19.0 software (IBM Corp.). Kaplan-Meier analysis and the log-rank test were used to calculate overall survival and disease-free survival of patients. A Cox proportional hazards model was used for multivariate survival analysis. Student's t-test and one-way analysis of variance followed by Tukey's post hoc test were used to compare the differences between two groups and $>2$ groups, respectively. $\mathrm{P}<0.05$ was considered to indicate a statistically significant difference.

\section{Results}

Expression of RAD18 is positively associated with poor prognosis in patients with $C C$. To investigate the potential role of RAD18 in the progression of CC, RAD18 expression was determined in $126 \mathrm{CC}$ tissues that were collected from Nanjing Medical University Affiliated Suzhou Hospital using immunohistochemistry (Fig. 1A). Kaplan-Meier analysis and log-rank tests indicated that patients with higher RAD18 expression 

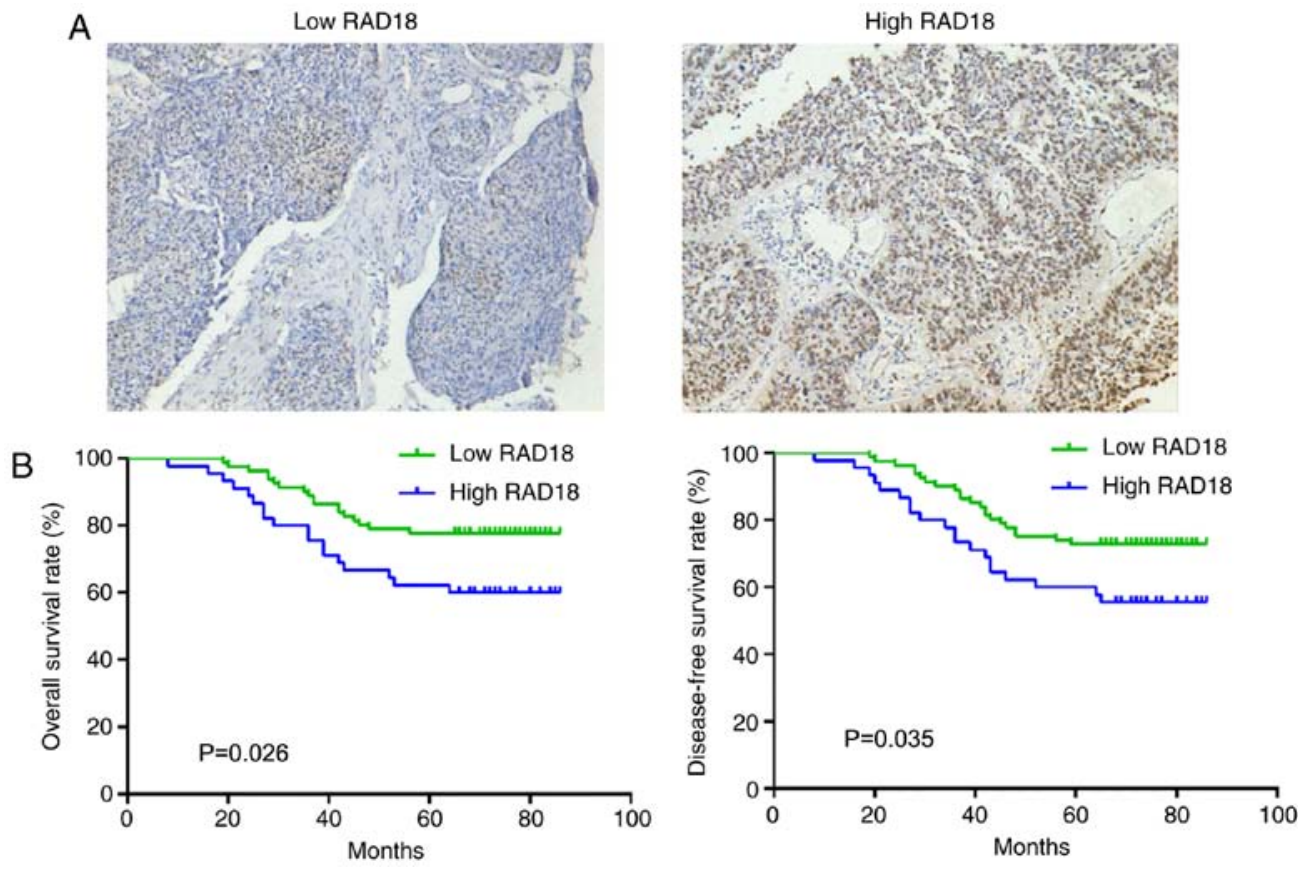

Figure 1. RAD18 expression is positively associated with the poor prognosis of patients with CC. (A) Immunohistochemical staining of RAD18 in 126 CC tissues (magnification, x100; Low expression=81, High expression=45). (B) Kaplan-Meier survival analysis of RAD18 expression and overall (P=0.026) or disease-free survival $(\mathrm{P}=0.035)$ of patients with $\mathrm{CC}$. $\mathrm{CC}$, cervical cancer.

$(\mathrm{n}=45)$ exhibited significantly shorter OS $(\mathrm{P}=0.026)$ and disease-free survival (DFS; $\mathrm{P}=0.035$ ) compared with patients with low RAD18 expression (n=81; Fig. 1B). Additionally, multivariate Cox regression analysis was performed to determine three of the main OS and DFS-associated factors. It was revealed that RAD18 was an independent prognostic marker for low OS (HR=2.125; 95\% $\mathrm{CI}=1.101-4.101 ; \mathrm{P}=0.025)$ and DFS (HR=2.031; 95\% CI=1.103-3.740; $\mathrm{P}=0.023$; Table III). Collectively, these results indicated that RAD18 may serve an important role in promoting the progression of CC.

RAD18 promotes $C C$ cell migration and invasion in vitro. To investigate how RAD18 regulates CC progression, the stable cell lines that RAD18 knockdown cell models were generated using RAD18-targeting shRNA. Western blotting was performed to detect changes in RAD18 expression (Fig. 2A). The results demonstrated that shRAD18 markedly suppressed RAD18 expression in HeLa229 and SiHa cells, indicating that the RAD18 knockdown cell models were successfully generated. As cell migration and invasion are important properties for cell metastasis (18), the cell lines were subjected to migration and invasion assays. Knockdown of RAD18 significantly inhibited the migratory and invasive abilities of HeLa229 and SiHa cells (Fig. 2B and C). In conclusions, these results suggested a potential role for RAD18 in CC cell invasion.

Differential gene expression in SiHa cells following RAD18 knockdown. To explore the potential mechanisms of the RAD18-mediated migration and invasion of CC cells, RNA-Seq transcriptome analysis was performed in SiHa-shRAD18 and SiHa-shNC cells. The data revealed that 349 genes exhibited a 1.5 -fold or higher change in expression in the RAD18 knockdown SiHa cells compared with the control group. Among them, 95 genes were upregulated and 254 genes were down-regulated (Fig. 3A). GO analysis revealed that the differentially expressed genes were involved in biological processes such as inflammatory and immune responses, exhibited molecular functions including chemokine activity, cytokine activity and receptor binding, and were distributed in extracellular space, extracellular regions, and the cell surface and cytoplasm (Fig. 3B). KEGG pathway analysis revealed that RAD18 knockdown altered the expression of genes associated with several key metastasis-associated signaling pathways, including NF-kB (19), nucleotide-binding oligomerization domain (NOD)-like receptor (20) and Toll-like receptor signaling pathways (21) (Fig. 3C).

RAD18 increases the migration and invasion of CC cells by promoting IL-1 $\beta$ expression. The expression levels of 10 representative genes were validated via RT-qPCR analysis, and those of baculoviral IAP repeat containing 3, C-X-C chemokine ligand (CXCL)8, IL-1 $\alpha$, IL-1 $\beta$ and intercellular adhesion molecule 1 were downregulated, consistent with microarray data, whereas STAT1, CXCL16 and CXCL2 were upregulated, the opposite of the microarray data (Fig. 3D). IL-1 $\beta$ is a pleiotropic cytokine that serves major roles in the regulation of immune and inflammatory responses (22). In addition, IL-1 participates in a wide range of processes, including regulation of the growth and metastasis of melanoma, glioma, meningioma, breast, cervical, thyroid and ovarian cancer cells (23). The mRNA and protein levels of IL-1 $\beta$ were further validated in SiHa-shRAD18 and SiHa-shNC cells using RT-qPCR and western blot analyses, with the observed results consistent with the previous analysis (Fig. 4A and B). To determine the potential role of IL-1 $\beta$ in RAD18-induced CC cell invasion, SiHa-shRAD18 cells were exposed to exogenous IL-1 $\beta$. As presented in Fig. 4C, IL-1 $\beta$ treatment rescued CC cell invasion in SiHa-shRAD18 cells, as determined by Transwell assays. 
Table III. Multivariate Cox proportional hazards analysis for candidate variables.

\begin{tabular}{|c|c|c|c|c|c|c|}
\hline \multirow[b]{2}{*}{ Variables } & \multicolumn{3}{|c|}{ Overall survival } & \multicolumn{3}{|c|}{ Disease-free survival } \\
\hline & HR & $95 \% \mathrm{CI}$ & P-value & HR & $95 \% \mathrm{CI}$ & P-value \\
\hline T stage & 12.646 & $4.210-37.983$ & $<0.001$ & 9.209 & $3.846-22.050$ & $<0.001$ \\
\hline $\mathrm{N}$ stage & 1.322 & $0.659-2.652$ & 0.433 & 1.091 & $0.559-2.129$ & 0.799 \\
\hline RAD18 (high vs. low) & 2.125 & $1.101-4.101$ & 0.025 & 2.031 & $1.103-3.740$ & 0.023 \\
\hline
\end{tabular}

CI, confidence interval; HR, hazard ratio.

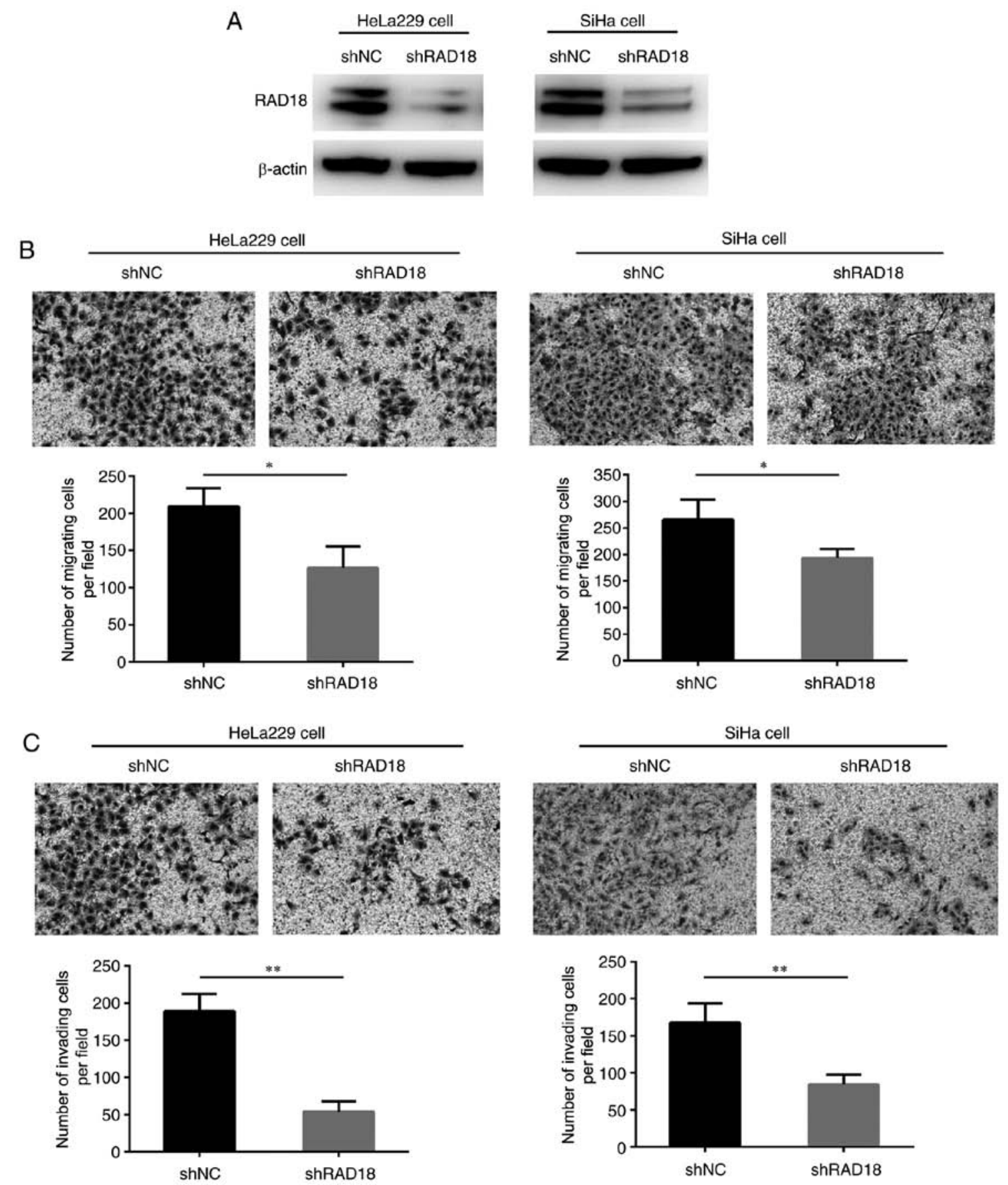

Figure 2. Knockdown of RAD18 inhibits CC cell migration and invasion in vitro. (A) Western blotting was performed to detect the RAD18 protein levels in $\mathrm{CC}$ cells transfected with a RAD18 shRNA lentivirus $(\mathrm{n}=3)$. (B) Transwell assays were performed to evaluate cell migration. RAD18 knockdown significantly reduced CC cell migration (magnification $x 100$ ). $\mathrm{N}=3$. ${ }^{*} \mathrm{P}<0.05$. (C) Matrigel Transwell assays were performed to evaluate cell invasion. RAD18 knockdown significantly suppressed the invasive abilities of cervical cancer cells (magnification $\mathrm{x} 100$ ). $\mathrm{N}=3 .{ }^{* *} \mathrm{P}<0.01$. CC, cervical cancer; sh, short hairpin RNA. 

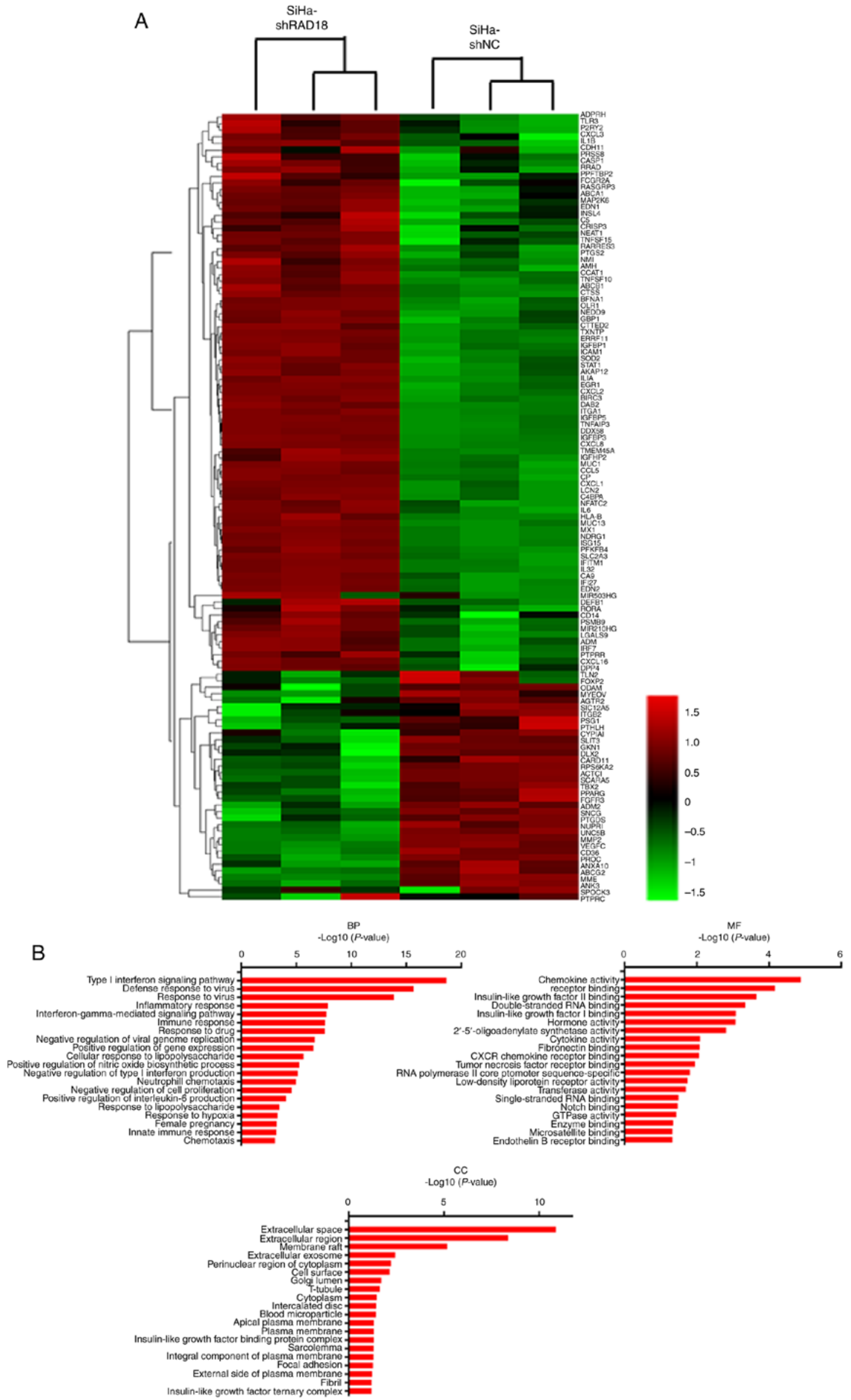

Figure 3. RAD18 regulatory network analysis. Differential gene expression in SiHa-shRAD18 cells. RNA isolated from RAD18 knockdown cells and control cells were analyzed by RNA sequencing. (A) Heat map of differentially expressed transcripts. (B) Gene Ontology based on all identified transcripts. 


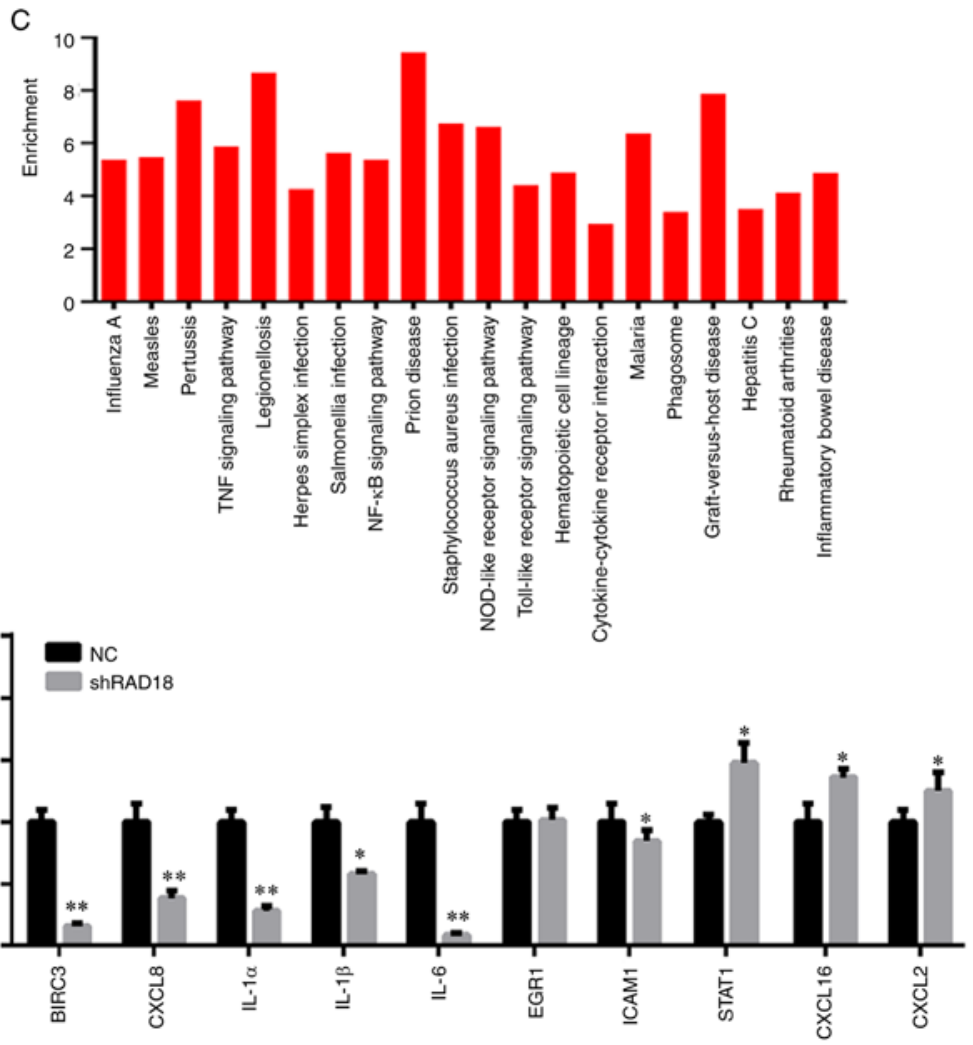

Figure 3. Continued. (C) Pathway analysis, based on all identified transcripts. (D) Differentially expressed transcripts were validated via reverse transcription-quantitative PCR analysis in SiHa-shRAD18 cells. $\mathrm{N}=3$. ${ }^{*} \mathrm{P}<0.05,{ }^{* *} \mathrm{P}<0.01$. BP, biological process; $\mathrm{CC}$, cellular component; MF, molecular function; sh, short hairpin RNA.

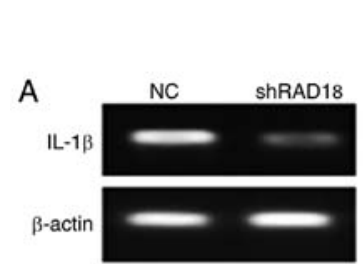

C

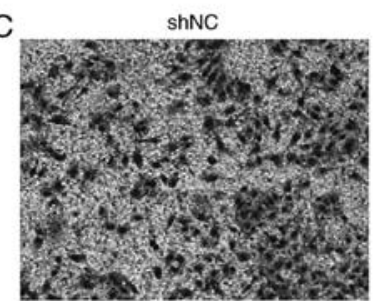

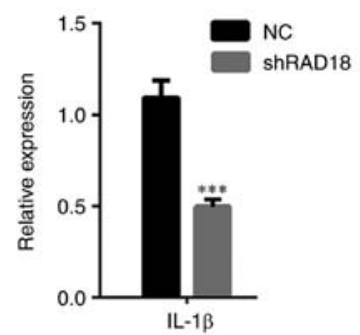

ShRAD18

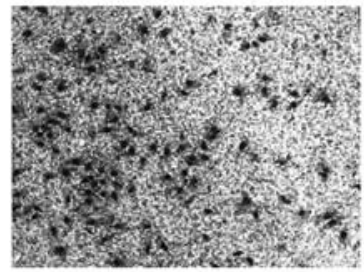

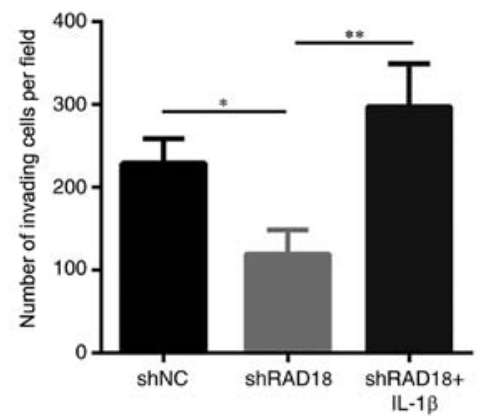

Figure 4. Exogenous IL-1 $\beta$ treatment rescues the invasion of cervical cancer cells following RAD18 knockdown. (A) Reverse transcription-quantitative PCR analysis was performed to determine IL-1 $\beta$ mRNA expression. The products of PCR were separated by electrophoresis on an agarose gel and stained with ethidium bromide to visualize the bands. $\mathrm{N}=3{ }^{* * *} \mathrm{P}<0.001$ (B) Western blot analysis were performed to validate the expression of IL-1 $\beta$ in SiHa-shRAD18 cells. (C) SiHa-shRAD18 cells were pretreated with IL-1 $\beta(10 \mathrm{ng} / \mathrm{ml})$ for $24 \mathrm{~h}$, and Transwell assays was performed to determine cell invasion (magnification $\mathrm{x} 100)$. $\mathrm{N}=3$. " $\mathrm{P}<0.05,{ }^{* *} \mathrm{P}<0.01$. sh, short hairpin RNA. 
Collectively, these results revealed that RAD18 enhanced the invasive abilities of $\mathrm{CC}$ cells, at least partially by regulating the expression of IL-1 $\beta$.

\section{Discussion}

In the present study, the expression of RAD18 was analyzed in $126 \mathrm{CC}$ samples, and it was found that the expression levels of RAD18 were negatively associated with the prognosis of patients with CC. High RAD18 expression was associated with reduced 5-year OS and DFS in patients, from 76.5 and $72.8 \%$, respectively, for patients with low expression to 62.2 and $60 \%$, respectively, for patients with high expression. Furthermore, multivariate Cox regression analysis revealed that high RAD18 expression may be an independent prognostic indicator of survival in patients with CC. Metastasis is the leading reason for the mortality of patients with cancer (24). The 5-year survival rate of patients with $\mathrm{CC}$ reduced from $80-95 \%$ in patients without lymph node metastases to $50-65 \%$ in patients with lymph nodes metastases (25). Using $\mathrm{CC}$ cell lines, it was demonstrated that knockdown of RAD18 significantly suppressed the invasiveness of CC cells in vitro (Fig. 2). Consistent with the present study, Shi et al (26) reported a positive association between positive expression of DNA pol $\zeta$, another TLS member, and the depth of the cervical stromal invasion of CC cells. They also observed that pol $\zeta$-positive expression was highly associated with the poor prognosis of patients with cervical squamous cell carcinoma that underwent adjuvant concurrent chemoradiation therapy following radical surgery treatment, indicating that pol $\zeta$ may be a potential biomarker of chemoradioresistance in CC (26). Yang et al (27) reported that protein reversionless 3-like (REV3L), the catalytic subunit of pol $\zeta$, also exhibited elevated expression in CC tissues compared with adjacent normal tissues. Furthermore, they found that overexpression of REV3L promoted chemoresistance in CC cells by inhibiting apoptosis.

To further investigate the molecular mechanisms underlying RAD18-mediated CC invasion, gene expression analysis was performed using an mRNA microarray, which identified 95 upregulated and 254 downregulated genes. Pathway analysis revealed that these genes were enriched in several metastasis-associated signaling pathways, including NF- $\kappa B$, NOD-like receptor and Toll-like receptor signal pathways (20). Although infection with human papilloma virus is considered to be the major event leading to $\mathrm{CC}$, viral infection alone is not sufficient for CC progression (28). A number of studies reported that chronic inflammation serves a crucial role in the development of various types of cancer, including colon cancer and CC $(29,30)$. As an important inflammatory cytokine, IL-1 $\alpha$ is produced by numerous types of cells, including macrophages, fibroblasts and cervical epithelium (31). Emerging evidence suggests that the secretion of IL-1 $\alpha$ within tumor microenvironments promotes the expression of a large number of growth factors, including IL-6 and vascular endothelial growth factor, in addition to the metastasis-associated MMPs $(32,33)$. MMPs are a group of enzyme that enhance the progression of the epithelial-to-mesenchymal transition $(34,35)$ by degrading various components of extracellular matrix proteins. Therefore, IL-1 has been proposed to facilitate the invasion and metastasis of cancers. Song et al (36) reported that IL-1 expression was associated with lymph node metastasis and stromal invasion in CC. IL-1 $\beta$ is a member of interleukin 1 family cytokines; the activation of IL-1 $\beta$ is involved in a variety of cellular process, including cell proliferation, differentiation and apoptosis (37). In the present study, it was demonstrated that RAD18 regulated the expression of IL-1 $\beta$ in CC. Furthermore, it was revealed that the exogenous IL- $1 \beta$ treatment can promote invasion in CC cells following RAD18 knockdown.

How RAD18 regulates the transcription of IL-1 $\beta$ remains unclear; however, it has been reported that the transcriptional factor NF- $\kappa$ B can transactivate IL- $1 \alpha$ and IL- $1 \beta$ following influenza virus infection (38). Zou et al (15) reported that RAD18 activated the JNK signal pathway, which is involved in the regulation of NF- $\mathrm{KB}$ transcriptional activity. The potential interaction of RAD18 with the JNK and NF- $\mathrm{KB}$ pathways requires further investigation.

Collectively, the present findings demonstrated that elevated RAD18 expression was positively associated with the poor prognosis of patients with CC. RAD18 promoted CC cell migration and invasion by upregulating IL-1 $\beta$. These data indicated that RAD18 may be a potential biomarker and/or therapeutic target in malignant $\mathrm{CC}$.

\section{Acknowledgements}

Not applicable

\section{Funding}

This work was supported by the National Natural Science Foundation of China (grant nos. 81672975 and 81802341), Jiangsu Provincial Medical Youth Talent (grant no. QNRC2016235), the Six Talent Peaks Project of Jiangsu Province of China (grant no. WSN095), the '333' Project of Jiangsu Province of China (grant no. BRA2016071), the Suzhou Administration of Science \& Technology (grant nos. SYS201567 and SS201649) and the Suzhou Key Medical Center (grant nos. SZZX201506 and SZZX201505), and the Ningbo Administration of Science \& Technology (grant no. 2017A610168).

\section{Availability of data and materials}

All data used or analyzed during the current study are included in this published article.

\section{Authors' contributions}

PL and SZ performed the majority of experiments and drafted the manuscript. JZ and ZS designed the research and assisted in drafting the manuscript. $\mathrm{CH}, \mathrm{AG}$ and $\mathrm{SH}$ contributed to data collection and interpretation, and critically reviewed the manuscript. All authors read and approved the final manuscript.

\section{Ethics approval and consent to participate}

The present study was approved by the Institutional Ethics Committee of the Nanjing Medical University. Informed 
consent was obtained from patients at the time of sample collection.

\section{Patient consent for publication}

Not applicable.

\section{Competing interests}

The authors declare that they have no competing interests.

\section{References}

1. Torre LA, Bray F, Siegel RL, Ferlay J, Lortet-Tieulent J and Jemal A: Global cancer statistics, 2012. CA Cancer J Clin 65: 87-108, 2015

2. Wang JY and Chen LJ: The role of miRNAs in the invasion and metastasis of cervical cancer. Biosci Rep 39: BSR20181377, 2019.

3. Oei AL, van Leeuwen CM, ten Cate R, Rodermond HM, Buist MR, Stalpers LJ, Crezee J, Kok HP, Medema JP and Franken NA: Hyperthermia selectively targets human papillomavirus in cervical tumors via p53-dependent apoptosis. Cancer Res 75: 5120-5129, 2015.

4. Thanapprapasr D, Nartthanarung A, Likittanasombut $\mathrm{P}, \mathrm{Na}$ Ayudhya NI,Charakorn C, Udomsubpayakul U, Subhadarbandhu T and Wilailak S: Bone metastasis in cervical cancer patients over a 10-year period. Int J Gynecol Cancer 20: 373-378, 2010.

5. Jackson SP and Bartek J: The DNA-damage response in human biology and disease. Nature 461: 1071-1078, 2009.

6. Ciccia A and Elledge SJ: The DNA damage response: Making it safe to play with knives. Mol Cell 40: 179-204, 2010.

7. Friedberg EC, Lehmann AR and Fuchs RP: Trading places: How do DNA polymerases switch during translesion DNA synthesis? Mol Cell 18: 499-505, 2005.

8. Ghosal G and Chen J: DNA damage tolerance: A double-edged sword guarding the genome. Transl Cancer Res 2: 107-129, 2013

9. Kannouche PL, Wing J and Lehmann AR: Interaction of human DNA polymerase eta with monoubiquitinated PCNA: A possible mechanism for the polymerase switch in response to DNA damage. Mol Cell 14: 491-500, 2004.

10. Bailly V, Prakash S and Prakash L: Domains required for dimerization of yeast Rad6 ubiquitin-conjugating enzyme and Rad18 DNA binding protein. Mol Cell Biol 17: 4536-4543, 1997.

11. Xin H, Lin W, Sumanasekera W, Zhang Y, Wu X and Wang Z: The human RAD18 gene product interacts with HHR6A and HHR6B. Nucleic Acids Res 28: 2847-2854, 2000.

12. Albertella MR, Lau A and O'Connor MJ: The overexpression of specialized DNA polymerases in cancer. DNA Repair (Amst) 4 583-593, 2005

13. Wong RP, Aguissa-Touré AH, Wani AA, Khosravi S, Martinka M and Li G: Elevated expression of Rad18 regulates melanoma cell proliferation. Pigment Cell Melanoma Res 25: 213-218, 2012

14. Zhou J, Zhang S, Xie L, Liu P, Xie F, Wu J, Cao J and Ding WQ: Overexpression of DNA polymerase iota (Poli) in esophageal squamous cell carcinoma. Cancer Sci 103: 1574-1579, 2012.

15. Zou S, Yang J, Guo J, Su Y, He C, Wu J, Yu L, Ding WQ and Zhou J: RAD18 promotes the migration and invasion of esophageal squamous cell cancer via the JNK-MMPs pathway. Cancer Lett 417: 65-74, 2018.

16. Kay FU, Kandathil A, Batra K, Saboo SS, Abbara S and Rajiah P: Revisions to the Tumor, Node, Metastasis staging of lung cancer (8th edition): Rationale, radiologic findings and clinical implications. World J Radiol 9: 269-279, 2017.

17. Livak KJ and Schmittgen TD: Analysis of relative gene expression data using real-time quantitative PCR and the 2(-Delta Delta C(T)) method. Methods 25: 402-408, 2001.

18. Robert J: Biology of cancer metastasis. Bull Cancer 100: 333-342, 2013.
19. Fusella F, Seclì L, Busso E, Krepelova A, Moiso E, Rocca S, Conti L, Annaratone L, Rubinetto C, Mello-Grand M, et al: The IKK/NF- $\kappa \mathrm{B}$ signaling pathway requires Morgana to drive breast cancer metastasis. Nat Commun 8: 1636, 2017.

20. Karki R, Man SM and Kanneganti TD: Inflammasomes and cancer. Cancer Immunol Res 5: 94-99, 2017.

21. Huang B, Zhao J, Unkeless JC, Feng ZH and Xiong H: TLR signaling by tumor and immune cells: A double-edged sword. Oncogene 27: 218-224, 2008.

22. Dinarello CA: Biologic basis for interleukin-1 in disease. Blood 87: 2095-2147, 1996.

23. Liu Q, Russell MR, Shahriari K, Jernigan DL, Lioni MI, Garcia FU and Fatatis A: Interleukin-1 $1 \beta$ promotes skeletal colonization and progression of metastatic prostate cancer cells with neuroendocrine features. Cancer Res 73: 3297-3305, 2013.

24. Wittekind C and Neid M. Cancer invasion and metastasis. Oncology 69 (Suppl 1): S14-S16, 2005.

25. Zhao S, Yao D, Chen J, Ding N and Ren F: MiR-20a promotes cervical cancer proliferation and metastasis in vitro and in vivo. PLoS One 10: e0120905, 2015.

26. Shi TY, Yang L, Yang G, Tu XY, Wu X, Cheng X and Wei Q: DNA polymerase $\zeta$ as a potential biomarker of chemoradiation resistance and poor prognosis for cervical cancer. Med Oncol 30: 500,2013

27. Yang L, Shi T, Liu F, Ren C, Wang Z, Li Y, Tu X, Yang G and Cheng $\mathrm{X}$ : REV3L, a promising target in regulating the chemosensitivity of cervical cancer cells. PLoS One 10: e0120334, 2015.

28. Goodman A: HPV testing as a screen for cervical cancer. BMJ 350: h2372, 2015.

29. Terzić J, Grivennikov S, Karin E and Karin M: Inflammation and colon cancer. Gastroenterology 138: 2101-2114, 2010.

30. Walch-Rückheim B, Mavrova R, Henning M, Vicinus B, Kim YJ, Bohle RM, Juhasz-Böss I, Solomayer EF and Smola S: Stromal fibroblasts induce CCL20 through IL6/C/EBP $\beta$ to support the recruitment of Th17 cells during cervical cancer progression. Cancer Res 75: 5248-5259, 2015.

31. Kelly PN, Romero DL, Yang Y, Shaffer AL III, Chaudhary D, Robinson S, Miao W, Rui L, Westlin WF, Kapeller R and Staudt LM: Selective interleukin-1 receptor-associated kinase 4 inhibitors for the treatment of autoimmune disorders and lymphoid malignancy. J Exp Med 212: 2189-2201, 2015.

32. Mar AC, Chu CH, Lee HJ, Chien CW, Cheng JJ, Yang SH, Jiang JK and Lee TC: Interleukin-1 receptor type 2 acts with c-Fos to enhance the expression of interleukin-6 and vascular endothelial growth factor a in colon cancer cells and induce angiogenesis. J Biol Chem 290: 22212-22224, 2015.

33. Kwon MJ, Hong E, Choi Y, Kang DH and Oh ES: Interleukin-1a promotes extracellular shedding of syndecan-2 via induction of matrix metalloproteinase-7 expression. Biochem Biophys Res Commun 446: 487-492, 2014

34. Ebelt ND, Cantrell MA and Van Den Berg CL: c-Jun N-terminal kinases mediate a wide range of targets in the metastatic cascade. Genes Cancer 4: 378-387, 2013.

35. van Zijl F, Krupitza G and Mikulits W: Initial steps of metastasis: Cell invasion and endothelial transmigration. Mutat Res 728: 23-34, 2011.

36. Song Z, Lin Y, Ye X, Feng C, Lu Y, Yang G and Dong C: Expression of IL-1 $\alpha$ and IL-6 is associated with progression and prognosis of human cervical cancer. Med Sci Monit 22: 4475-4481, 2016.

37. Niebler M, Qian X, Höfler D, Kogosov V, Kaewprag J, Kaufmann AM, Ly R, Böhmer G, Zawatzky R, Rösl F and Rincon-Orozco B: Post-translational control of IL-1 $\beta$ via the human papillomavirus type 16 E6 oncoprotein: A novel mechanism of innate immune escape mediated by the E3-ubiquitin ligase E6-AP and p53. PLoS Pathog 9: e1003536, 2013.

38. Pahl HL and Baeuerle PA: Expression of influenza virus hemagglutinin activates transcription factor NF-kappa B. J Virol 69: 1480-1484, 1995 